J. Lundén, V. Koivunen, A. Huttunen and H. V. Poor, "Censoring for collaborative spectrum sensing in cognitive radios," in Proceedings of the 41st Asilomar Conference on Signals, Systems, and Computers, Pacific Grove, CA, USA, November 4-7, 2007, pp. 772-776.

(C) 2007 IEEE. Reprinted with permission.

This material is posted here with permission of the IEEE. Such permission of the IEEE does not in any way imply IEEE endorsement of any of the Helsinki University of Technology's products or services. Internal or personal use of this material is permitted. However, permission to reprint/republish this material for advertising or promotional purposes or for creating new collective works for resale or redistribution must be obtained from the IEEE by writing to pubs-permissions@ieee.org.

By choosing to view this material, you agree to all provisions of the copyright laws protecting it. 


\title{
Censoring for Collaborative Spectrum Sensing in Cognitive Radios
}

\author{
Jarmo Lundén*, Visa Koivunen* ${ }^{* \ddagger}$, Anu Huttunen ${ }^{\dagger}$ and H. Vincent Poor ${ }^{\ddagger}$ \\ *SMARAD CoE, Signal Processing Laboratory \\ Helsinki Univ. of Technology, Finland \\ Email: \{jrlunden,visa\}@wooster.hut.fi \\ ${ }^{\dagger}$ Nokia Research Center, Helsinki, Finland \\ Email: anu.huttunen@nokia.com \\ ${ }^{\ddagger}$ School of Engineering and Applied Science, \\ Princeton University \\ Email: poor@princeton.edu
}

\begin{abstract}
Cooperative spectrum sensing among multiple cognitive radios mitigates the effects of shadowing and fading. However, it also generates overhead traffic which consumes more power in battery operated mobile terminals. In this paper a censoring scheme for spectrum sensing is proposed. Only informative test statistics are transmitted to the fusion center or shared with other secondary users. Two cooperative censoring test statistics based on cyclostationarity are proposed. Constant false alarm rate tests are derived and asymptotic distributions of test statistics established. The asymptotic distributions are approximated using characteristic functions. Limits for the censoring (no-send) region are derived. The performance of the proposed censoring scheme is illustrated through simulations in a multipath radio environment. Only a minor performance loss is experienced in comparison to uncensored cooperative detection even under very strict constaints on communication rates for the secondary users.
\end{abstract}

\section{INTRODUCTION}

Cognitive radios have been suggested as an enabling tool for dynamic allocation of radio spectrum. Cognitive radios sense the radio spectrum in order to find opportunities for agile spectrum use. Furthermore, spectrum sensing is critical for managing the level of interference caused to the primary users of the spectrum. Through sensing cognitive radios acquire information about the radio operating environment. This enables the cognitive radio to adjust its operating parameters, such as carrier frequency, transmit power, and waveforms dynamically in order to provide the best available connection to meet the user's needs within the constraints on interference.

Primary user transmissions must be detected reliably even in the low signal-to-noise ratio (SNR) regime and in the face of shadowing and fading. Communication signals are typically cyclostationary, and have many periodic statistical properties related, e.g., to the symbol rate, the coding and modulation schemes as well as the guard periods. These properties can

Jarmo Lundén's work was supported by GETA graduate school, Finnish Defence Forces Technical Research Centre and Nokia Foundation.

The funding for Visa Koivunen's sabbatical term at Princeton University was provided by the Academy of Finland.

H. Vincent Poor's work was supported by the US National Science Foundation under Grants ANI-03-38807 and CNS-06-25637. be exploited in designing a detector for the presence of signals having multiple cyclic frequencies [1]. Such a detector exploits the rich structure present in communication signals to improve the detector performance. Moreover, it allows for distinguishing between the primary and secondary users' signals. The cyclostationarity test introduced in [1] extends the method of [2] to take into account the rich information present at different cyclic frequencies.

Cooperation among the cognitive radio terminals allows for mitigating the effects of shadowing and fading through diversity, improves the detector performance and provides a larger footprint for the cognitive radio system. Collaborative detection is performed here by combining the quantized local test statistics from many secondary users. In order to optimize the performance under constraints on the amount of data each secondary user is allowed to send, we propose a censoring approach for the collaborative spectrum sensing. Two different techniques for censoring the generalized likelihood ratios (GLRs) from the cyclostationarity based test are proposed. The censoring scheme is applicable for other type of local test statistics, too. In censoring, the cognitive radios send only the informative test statistics to the fusion center (FC) or share it with other users in a decentralized system with no FC. Otherwise, the terminals do not send their test statistics or local decisions. Typically a very large or a very small value of the test statistic is considered to be informative. The boundaries of the no-send region for the secondary users are optimized under constraints on data rate and false alarm probabilities. Censoring facilitates saving energy in battery operated cognitive radio terminals because the transmitter needs to be used less frequently. Censoring has been employed in energy efficient sensor networks in [3], [4], [5].

This paper is organized as follows. In Section II, the statistical tests for multiple cyclic frequencies and their statistical properties are briefly described. The censoring approach for collaborative spectrum sensing is introduced in Section III. The limits for the no-send region are derived and approximations to the asymptotic distributions of the test statistics are derived using a Fourier-series method that numerically 
inverts the characteristic functions. In Section IV, simulation examples demonstrate the resulting performance gains in the low SNR regime and the benefits of cooperative detection under constraints on transmission rate. Finally, Section V concludes the paper.

\section{Detection USING MULTIPLE CYCLIC FREQUENCIES}

In the following we briefly present the test for multiple cyclic frequencies [1]. It is an extension of the test for a single cyclic frequency in [2].

In order to test for the presence of second-order cyclostationarity at any of the cyclic frequencies of interest $\alpha \in \mathcal{A}$ simultaneously, the hypothesis testing problem is formulated as follows:

$$
\begin{aligned}
& H_{0}: \forall \alpha \in \mathcal{A} \text { and } \forall\left\{\tau_{n}\right\}_{n=1}^{N} \Longrightarrow \hat{\boldsymbol{r}}_{x x^{(*)}}(\alpha)=\boldsymbol{\epsilon}_{x x^{(*)}}(\alpha) \\
& H_{1}: \text { for some } \alpha \in \mathcal{A} \text { and for some }\left\{\tau_{n}\right\}_{n=1}^{N} \\
& \quad \Longrightarrow \hat{\boldsymbol{r}}_{x x^{(*)}}(\alpha)=\boldsymbol{r}_{x x^{(*)}}(\alpha)+\boldsymbol{\epsilon}_{x x^{(*)}}(\alpha) .
\end{aligned}
$$

In the above expression, $x$ is the received signal and $\hat{\boldsymbol{r}}_{x x^{(*)}}(\alpha)$ ((*) denotes an optional complex conjugation) is a $1 \times 2 N$ vector containing the real and imaginary parts of the estimated cyclic covariances at different lags $\tau_{n}$ ( $N$ lags in total) at the candidate cyclic frequency $\alpha$ stacked in the same vector, i.e.,

$$
\begin{array}{r}
\hat{\boldsymbol{r}}_{x x^{(*)}}(\alpha)=\left[\operatorname{Re}\left\{\hat{R}_{x x^{(*)}}\left(\alpha, \tau_{1}\right)\right\}, \ldots, \operatorname{Re}\left\{\hat{R}_{x x^{(*)}}\left(\alpha, \tau_{N}\right)\right\},\right. \\
\left.\operatorname{Im}\left\{\hat{R}_{x x^{(*)}}\left(\alpha, \tau_{1}\right)\right\}, \ldots, \operatorname{Im}\left\{\hat{R}_{x x^{(*)}}\left(\alpha, \tau_{N}\right)\right\}\right]
\end{array}
$$

where $\hat{R}_{x x^{(*)}}(\alpha, \tau)$ is an estimate of the (conjugate) cyclic autocorrelation that may be obtained using $M$ observations as

$$
\hat{R}_{x x^{(*)}}(\alpha, \tau)=\frac{1}{M} \sum_{t=1}^{M} x(t) x^{(*)}(t+\tau) e^{-j 2 \pi \alpha t} .
$$

$\boldsymbol{\epsilon}_{x x^{(*)}}$ denotes the estimation error which is assumed to be asymptotically normally distributed with a variance approaching zero as $M$ goes to infinity [2].

For this detection problem, we have the following two test statistics [1]:

$$
\begin{aligned}
\mathcal{D}_{m, L} & =\max _{\alpha \in \mathcal{A}} \sum_{i=1}^{L} \mathcal{T}_{x x^{(*)}}^{(i)}(\alpha) \\
\mathcal{D}_{s, L} & =\sum_{i=1}^{L} \sum_{\alpha \in \mathcal{A}} \mathcal{T}_{x x^{(*)}}^{(i)}(\alpha)
\end{aligned}
$$

where $L$ is the number cooperating secondary users and $\mathcal{T}_{x x^{(*)}}^{(i)}(\alpha)$ denotes the cyclostationarity based test statistic from the $i^{\text {th }}$ secondary user:

$$
\mathcal{T}_{x x^{(*)}}(\alpha)=M \hat{\boldsymbol{r}}_{x x^{(*)}} \hat{\boldsymbol{\Sigma}}_{x x^{(*)}}^{-1} \hat{\boldsymbol{r}}_{x x^{(*)}}^{T} .
$$

$\boldsymbol{\Sigma}_{x x^{(*)}}$ is the $2 N \times 2 N$ asymptotic covariance matrix of $\hat{\boldsymbol{r}}_{x x^{(*)}}$ that can be calculated as [2]

$$
\boldsymbol{\Sigma}_{x x^{(*)}}(\alpha)=\left[\begin{array}{ll}
\operatorname{Re}\left\{\frac{\boldsymbol{Q}+\boldsymbol{Q}^{*}}{2}\right\} & \operatorname{Im}\left\{\frac{\boldsymbol{Q}-\boldsymbol{Q}^{*}}{2}\right\} \\
\operatorname{Im}\left\{\frac{\boldsymbol{Q}+\boldsymbol{Q}^{*}}{2}\right\} & \operatorname{Re}\left\{\frac{\boldsymbol{Q}^{*}-\boldsymbol{Q}}{2}\right\}
\end{array}\right]
$$

where the $(m, n)$ th entries of the covariance matrices $\boldsymbol{Q}$ and $Q^{*}$ are given by

$$
\begin{aligned}
\boldsymbol{Q}(m, n) & =S_{f_{\tau_{m}} f_{\tau_{n}}}(2 \alpha, \alpha) \\
\boldsymbol{Q}^{*}(m, n) & =S_{f_{\tau_{m}} f_{\tau_{n}}}(0,-\alpha) .
\end{aligned}
$$

$S_{f_{\tau_{m}} f_{\tau_{n}}}(\alpha, \omega)$ and $S_{f_{\tau_{m}} f_{\tau_{n}}}^{*}(\alpha, \omega)$ denote the unconjugated and conjugated cyclic spectra of $f(t, \tau)=x(t) x^{(*)}(t+\tau)$, respectively, that can be estimated using, e.g., frequency smoothed cyclic periodograms as

$$
\begin{aligned}
\hat{S}_{f_{\tau_{m}} f_{\tau_{n}}}(2 \alpha, \alpha)= & \frac{1}{M T} \sum_{s=-(T-1) / 2}^{(T-1) / 2} W(s) \\
& \cdot F_{\tau_{n}}\left(\alpha-\frac{2 \pi s}{M}\right) F_{\tau_{m}}\left(\alpha+\frac{2 \pi s}{M}\right) \\
\hat{S}_{f_{\tau_{m}} f_{\tau_{n}}}^{*}(0,-\alpha)= & \frac{1}{M T} \sum_{s=-(T-1) / 2}^{(T-1) / 2} W(s) \\
& \cdot F_{\tau_{n}}^{*}\left(\alpha+\frac{2 \pi s}{M}\right) F_{\tau_{m}}\left(\alpha+\frac{2 \pi s}{M}\right)
\end{aligned}
$$

where $F_{\tau}(\omega)=\sum_{t=1}^{M} x(t) x^{(*)}(t+\tau) e^{-j \omega t}$ and $W$ is a normalized spectral window of odd length $T$.

The relationship of the above test statistics to the GLR is established in [1]. The asymptotic properties of the test statistics under the null hypothesis required for the constant false alarm rate (CFAR) tests are summarized next.

The cyclostationary test statistic $\mathcal{T}_{x x^{(*)}}(\alpha)$ is under the null hypothesis asymptotically $\chi_{2 N}^{2}$ distributed [2].

The asymptotic cumulative distribution function of the test statistic $\mathcal{D}_{m, L}$ under the null hypothesis is given by [1]

$$
F\left(y, 2 N L, N_{\alpha}\right)=\left(1-e^{-y / 2} \sum_{n=0}^{N L-1} \frac{(y / 2)^{n}}{n !}\right)^{N_{\alpha}}, y>0 .
$$

$N_{\alpha}$ is the number of cyclic frequencies in set $\mathcal{A}$.

Finally, the asymptotic distribution of $\mathcal{D}_{s, L}$ is under the null hypothesis $\chi_{2 N N_{\alpha} L}^{2}[1]$.

In the next section collaborative detection with censoring will be introduced. Only the sum statistic of the multicycle test statistics will be considered since in [1] it was found to have sligthly better performance. Moreover, the maximum test statistic $\mathcal{D}_{m, L}$ generates $N_{\alpha}$ times more data traffic than the sum test statistic $\mathcal{D}_{s, L}$ if the maximization over the cyclic frequencies is performed at the FC.

\section{Collaborative Detection with CEnsoring}

The secondary users transmit data containing the spectrum sensing results to a Fusion Center (FC) or share it with other users. The amount of data each secondary user is allowed to communicate may be strictly constrained. Thus, only the relevant or informative test statistics should be transmitted to the FC or the other users. This operation is called censoring. It reduces the energy consumption since fewer terminals are transmitting. In this paper we propose two censoring strategies for spectrum sensing and study their performance under 
constraints on data rate and false alarm probabilities. The total number of collaborating users is denoted by $L$. The first test uses the following test statistic:

$$
\mathcal{D}_{K}=\sum_{i=1}^{K} \sum_{\alpha \in \mathcal{A}} \mathcal{T}_{x x^{(*)}}^{(i)}(\alpha),
$$

where $\mathcal{A}$ is the set of cyclic frequencies of interest and $K$ out of $L$ users transmit their test statistics, i.e., do not perform censoring.

The idea in the above test is that users whose test statistic value remains within the censoring region do not send their statistics. In the second test, the test statistics of the users not transmitting their test statistics are replaced by an estimate of their likelihood ratios. That is, the second test uses the following test statistic:

$$
\mathcal{D}_{L}=\mathcal{D}_{K}+\sum_{i=1}^{L-K} d_{i}=\sum_{i=1}^{K} \sum_{\alpha \in \mathcal{A}} \mathcal{T}_{x x^{(*)}}^{(i)}(\alpha)+\sum_{i=1}^{L-K} d_{i}
$$

where $d_{i}$ is the average of the local generalized log-likelihood ratio of the $i$ th user (i.e., the test statistic $\sum_{\alpha \in \mathcal{A}} \mathcal{T}_{x x^{(*)}}^{(i)}(\alpha)$ ) in the no-send region. That is, the latter sum corresponds to the likelihood ratios at the no-send region. Under the null hypothesis the values for $d_{i}$ are obtained by calculating the mean values of $\chi_{2 N N_{\alpha}}^{2}$ distributed random variables limited to the no-send regions.

Censoring affects the distribution of the global test statistics at the FC or node where the statistics are combined. We can approximate the asymptotic distributions numerically using the characteristic functions of the test statistics. In the following we derive the characteristic functions of the above test statistics under the null hypothesis. For simplicity we assume that $\kappa=\kappa_{i}, \forall i$. The results can be extended to accommodate different communication rate constraints among the secondary users. We will first derive the characteristic function of $\mathcal{D}_{K}$. The characteristic function of $\mathcal{D}_{L}$ can be easily obtained from the characteristic function of $\mathcal{D}_{K}$ as will be shown later.

The distribution of the test statistic $\mathcal{D}_{K}$ can be defined using the conditional distributions as (similar equation can be written for $\mathcal{D}_{L}$ as well)

$$
p\left(\mathcal{D}_{K} \mid H_{0}\right)=\sum_{k=0}^{L} p\left(\mathcal{D}_{K} \mid K=k, H_{0}\right) p\left(K=k \mid H_{0}\right),
$$

where the probabilities of different values of $K$ are given by

$$
p\left(K=k \mid H_{0}\right)=\left(\begin{array}{l}
L \\
k
\end{array}\right) \kappa^{k}(1-\kappa)^{L-k} .
$$

The probability density function (pdf) of $\mathcal{D}_{K=1}$ (i.e., for $k=1$ ) is given by a truncated chi-square pdf, i.e.,

$g\left(y, 2 N \mid K=1, H_{0}\right)=\frac{1}{1-G(t)} \cdot \frac{1}{2^{N} \Gamma(N)} y^{N-1} e^{-y / 2}, y \geq t$

where $2 N$ is the number of degrees of freedom and $\Gamma(\cdot)$ denotes the gamma function. The censoring threshold is denoted by $t$ (for the reasons explained later the censoring region is a single interval with a lower threshold of 0 ) and $G(\cdot)$ denotes the cumulative distribution function of the chi-square distribution. For $y<t, g\left(y, 2 N \mid K=1, H_{0}\right)=0$. Next the characteric function of a single censored test statistic is derived.

The characteristic function of a random variable $Y$ always exists and it is defined by the following expectation

$$
\Phi(\omega)=E[\exp (j \omega y)]
$$

where $j$ denotes the imaginary unit. Hence, for the single censored test statistic the characteric function is defined by

$$
\Phi_{\mathcal{T}}(\omega)=\int_{t}^{\infty} \exp (j \omega y) g\left(y, 2 N \mid k=1, H_{0}\right) d y .
$$

Using repeated integration by parts the following result is obtained

$$
\begin{aligned}
\Phi_{\mathcal{T}}(\omega)= & \frac{1}{1-G(t)} \sum_{n=1}^{N}\left(\frac{1}{(N-n) !} 2^{-N+n} t^{N-n}\right. \\
& \left.\cdot(1-2 j \omega)^{-n} \exp (-(1-2 j \omega) t / 2)\right) .
\end{aligned}
$$

Since the individual test statistics $\mathcal{T}_{x x^{(*)}}^{(i)}$ are independent, the characteristic function of $\mathcal{D}_{k}$ factors to a product of the characteristic functions of the individual test statistics. That is,

$$
\Phi_{\mathcal{D}_{k}}(\omega)=\prod_{i=1}^{k} \Phi_{\mathcal{T}_{i}}(\omega)=\Phi_{\mathcal{T}}(\omega)^{k}
$$

Now the characteristic function of $\mathcal{D}_{L}$ for a given $K=k$ is given by $\Phi_{\mathcal{D}_{L}}(\omega)=\exp (j \omega(L-k) d) \Phi_{\mathcal{D}_{k}}(\omega)$ where $d=$ $d_{i}, \forall i$. This follows directly from (18) since $d$ is non-random.

The characteristic function uniquely defines the distribution of a random variable. Consequently, the distributions $p\left(\mathcal{D}_{K} \mid K=k, H_{0}\right)$ and $p\left(\mathcal{D}_{L} \mid K=k, H_{0}\right)$ can be approximated by numerically inverting the characteristic function. Here we employ one of the most straightforward and simplest Fourier-series methods for numerical inversion of the characteristic function, the Method A of Bohman [6]. There exists many more sophisticated and accurate methods but the accuracy of the chosen method is more than sufficient for the application at hand.

Using the Method A of Bohman, the value of cumulative distribution function $G(y)$ of a random variable $Y$ with zero mean and unit variance is approximated by

$$
G(y) \approx \frac{1}{2}+\frac{\eta y}{2 \pi}-\sum_{\substack{\nu=1-H \\ \nu \neq 0}}^{H-1} \frac{\Phi_{Y}(\eta \nu)}{2 \pi j \nu} e^{-j \eta \nu y},
$$

where $\Phi_{Y}(\cdot)$ is the characteristic function of $Y . H$ defines the number of points $2 \mathrm{H}-1$ at which the distribution is estimated and $\eta$ is a constant chosen such that the full range of the distribution is represented (i.e., includes both 0 and 1). In case the points $y$ are chosen as the Fourier frequencies, i.e., $y_{k}=$ $2 \pi(k-H) /(2 \eta(H-1)), k=1, \ldots, 2 H-1$, and care is taken to exclude the undefined value for index $\nu=0$ the sum in (22) can be calculated using the Fast-Fourier transform (FFT). 
Equation (22) is defined for a normalized random variable with a mean zero and unit variance. Thus, the test statistics have to be normalized as well. The mean and variance can be easily calculated by differentiating the characteristic function since $\Phi_{Y}^{(n)}(0)=j^{n} E\left[y^{n}\right]$ and the variance $\sigma^{2}=E\left[y^{2}\right]-E[y]^{2}$. Finally, the characteristic function of a normalized variable $Z=(Y-\mu) / \sigma$ is given by $\Phi_{Z}(\omega)=$ $\exp (-j \omega \mu / \sigma) \Phi_{Y}(\omega / \sigma)$.

Using (22) the distributions $p\left(\mathcal{D}_{k} \mid K=k, H_{0}\right)$ and $p\left(\mathcal{D}_{L} \mid K=k, H_{0}\right)$ can be approximated. The combined distributions $p\left(\mathcal{D}_{K} \mid H_{0}\right)$ and $p\left(\mathcal{D}_{L} \mid H_{0}\right)$ are obtained by multiplying the conditional distributions with the probabilities of different values of $K$ in (16), respectively. The distribution values between the FFT points can be interpolated.

In order to obtain a desired false alarm rate $p_{f a}$, a single test threshold $\gamma$ may be set using the following equations

$$
p_{f a}=p\left(\mathcal{D}_{K}>\gamma \mid H_{0}\right) \quad \text { or } \quad p_{f a}=p\left(\mathcal{D}_{L}>\gamma \mid H_{0}\right) .
$$

Alternatively, different thresholds may be used for different values of number of received test statistics $K$. The desired false alarm rate is obtained if the thresholds $\gamma_{k}, k=1, \ldots, L$, satisfy the following condition (similarly for $\mathcal{D}_{L}$ )

$$
p_{f a}=\sum_{k=1}^{L} p\left(\mathcal{D}_{K}>\gamma_{k} \mid K=k, H_{0}\right) p\left(K=k \mid H_{0}\right) .
$$

In the above expression, it is assumed that if none of the users transmits the decision is always $H_{0}$. For example, the thresholds $\gamma_{k}$ may be chosen such that $p\left(\mathcal{D}_{K}>\gamma_{k} \mid K=\right.$ $\left.k, H_{0}\right)=p_{f a} / \sum_{k=1}^{L} p\left(K=k \mid H_{0}\right), \forall k$.

The remaining task is to find the censoring regions for the collaborating secondary users under the constraints on the data rate and false alarm probability. Here we adopt the strategy suggested in [4] where each user is assigned a separate communication rate constraint of the form

$$
p\left(\sum_{\alpha \in \mathcal{A}} \mathcal{T}_{x x^{(*)}}^{(i)}(\alpha)>t_{2, i} \mid H_{0}\right) \leq \kappa_{i},
$$

where $\kappa_{i} \leq 1$ is the send rate of user $i$ and $t_{2, i}$ is the upper limit of the censoring region of the user $i$. This type of constraint is natural in a scenario where the secondary user terminals may have very different capabilities for data transmission. However, in this work as already stated earlier, the constraints on data transmission are assumed to be the same for all secondary users. The lower limit is chosen to be $t_{1, i}=0, \forall i$. This choice minimizes the probability of missed detection for any false-alarm constraint less than or equal to $1-\prod_{i=1}^{L}\left(1-\kappa_{i}\right)$ when the censoring region is a single interval of the likelihood ratio [4]. The asymptotic distribution of the test statistic in (25) is under the null hypothesis $\chi_{2 N N_{\alpha}}^{2}$. Thus, the threshold values $t_{2, i}$ needed to meet the communication rate constraints can easily be selected independently by the secondary users. However, the threshold values must be communicated to the FC or to the secondary user making the global decision.

\section{Simulation ExAmples}

The performance of the detectors with censoring for the test statistics in (13) and (14) were tested using one and two cyclic frequencies. The number of collaborating users was taken to be $L=10$. Values $\eta=0.5$ and $H=1000$ were used to approximate the asymptotic distributions.

The primary user signal was an orthogonal frequency division multiplex (OFDM) signal

$$
x(t)=\sum_{n=0}^{N_{c}-1} \sum_{l=-\infty}^{\infty} c_{n, l} g\left(t-l T_{s}\right) e^{j\left(2 \pi / N_{c}\right) n\left(t-l T_{s}\right)}
$$

where $N_{c}$ is the number of subcarriers, $T_{s}$ is the symbol length, $g(t)$ denotes the rectangular pulse of length $T_{s}$, and $c_{n, l}$ 's denote the data symbols. The symbol length is the sum of the length of the useful symbol data $T_{d}$ and the length of the cyclic prefix $T_{c p}$, i.e., $T_{s}=T_{d}+T_{c p}$. The OFDM signal exhibits cyclostationarity at the integer multiples of the symbol rate $\alpha=k / T_{s}, k=0, \pm 1, \pm 2, \ldots$. The cyclic frequencies used in the calculation of the test statistics correspond to $1 / T_{s}$ or $1 / T_{s}$ and $2 / T_{s}$ in the case of one or two cyclic frequencies, respectively. The test statistics were calculated using two time lags $\pm T_{d}$. That is, the detectors assume the knowledge of the symbol frequency and the useful symbol length.

In the simulations the 3GPP typical urban channel model TUx $(\Delta t=32.55 n s)$ was used with mobile speed of 3 $\mathrm{km} / \mathrm{h}$ [7]. The SNR is defined as $\mathrm{SNR}=10 \log _{10} \frac{\sigma_{x}^{2}}{\sigma_{n}^{2}}$, where $\sigma_{x}^{2}$ and $\sigma_{n}^{2}$ are the variances of the signal and the noise, respectively. The channel and AWG noise experienced by each secondary user were different. The cyclic spectrum estimates were calculated using a length 2049 Kaiser window with $\beta$ parameter of 10 . FFT was employed for fast calculation. The FFT size was $2^{14}=16384$. The OFDM signal had 32 subcarriers and the length of the cyclic prefix was $1 / 4$ of the useful symbol data. 16-QAM modulation was used for each subcarrier. The carrier frequency was $2.5 \mathrm{GHz}$. Subcarrier spacing was $15 \mathrm{kHz}$. The signal length was 100 OFDM symbols. The signal was not oversampled. The simulations were averaged over 2000 experiments.

The detection probability as a function of SNR for a constant false alarm rate of 0.05 is shown in Fig. 1. The censoring thresholds were set according to the communication rate constraints $\kappa_{i}=0.1, \forall i$. The detection probability for 1,3 , and 10 secondary users without censoring is shown for comparison for the case of two cyclic frequencies. In all the simulations the sum test statistic in (5) has been used for the non-censoring cases. Close to similar performance to the 10 user case, and significantly better than in the 1 and 3 user cases, is obtained with the censoring schemes under strict constraints on transmission rates. It can be seen that the performance of the test statistic $\mathcal{D}_{L}$ is better than the performance of $\mathcal{D}_{K}$. The detection probability is higher in all the cases when two cyclic frequencies are used as compared to the case when only one cyclic frequency is used.

The number of users $K$ transmitting their test statistic to the FC in the censoring case is shown as a function of the SNR 


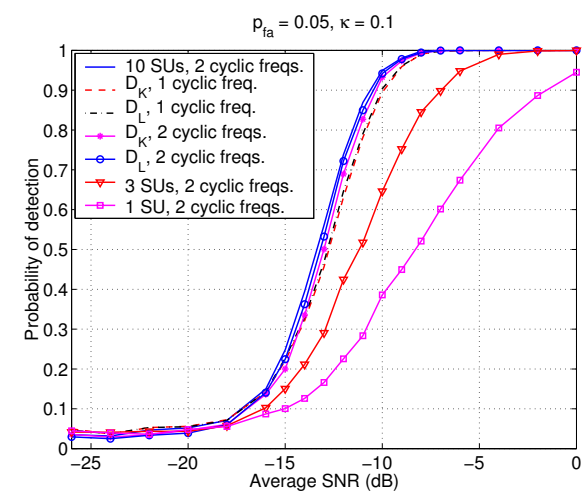

Figure 1. Probability of detection vs. SNR. Close to similar performance to the 10 user case, and significantly better than in the 1 and 3 user cases, is obtained with the censoring schemes under strict constraints on transmission rates. Using multiple frequencies improves performance further. The test statistic $\mathcal{D}_{L}$ outperforms the test statistic $\mathcal{D}_{K}$.

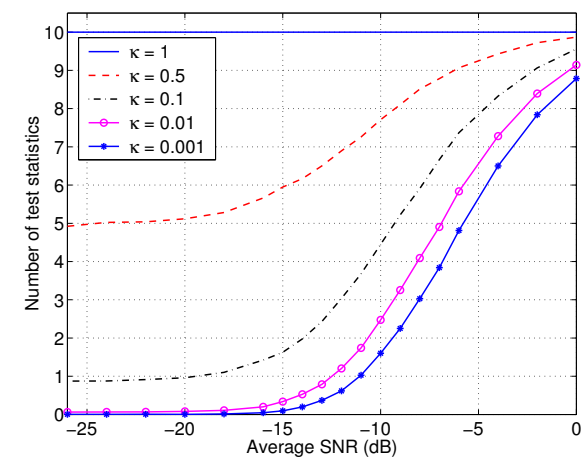

Figure 2. Average number of users transmitting the test statistic to the FC vs. SNR for the detectors based on two cyclic frequencies for different communication rate constraints.

in Fig. 2 for the case of two cyclic frequencies for different communication rate constraints. Note that both test statistics $\mathcal{D}_{L}$ and $\mathcal{D}_{K}$ use the same number of users due to the same communication rate constraint. E.g., for $\kappa=0.1$ in the low SNR regime approximately one of the users is transmitting the test statistic which corresponds to the communications constraint imposed on the users.

Fig. 3 illustrates the performance of the censoring test based on the test statistic $\mathcal{D}_{L}$ using two cyclic frequencies for different communication rate constraints for a constant false alarm rate of 0.01 . It can be seen that the performance loss due to censoring is minor even for very strict communication rate constraints.

\section{CONCLUSION}

In this paper a collaborative spectrum sensing scheme based on censoring of the uninformative test statistics has been proposed. The idea of this scheme is that users whose test statistics are not informative enough refrain from transmitting them to the FC or to the secondary user making the decision. This reduces considerably the amount of data transmitted. Consequently, the scheme provides energy efficiency which

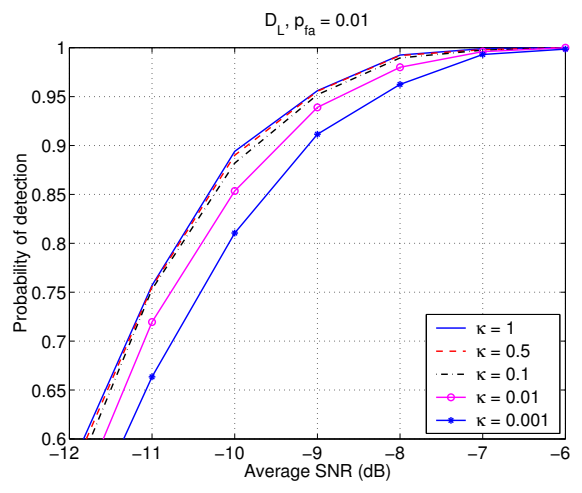

Figure 3. Probability of detection vs. SNR for different communication rate constraints for the $\mathcal{D}_{L}$ test statistic using two cyclic frequencies. The performance loss due to censoring is relatively small even for very strict communication rate constraints.

is important, especially, in mobile applications where the terminals are battery operated.

Two censoring test statistics based on cyclostationarity have been proposed. Their asymptotic distributions have been established using numerical inversion of the characteristic functions. Simulations have demonstrated that the proposed censoring strategies provide almost equal performance in comparison to no censoring scheme. Multipath environments under strict constraints on communication rates have been considered. We have seen that the performance loss due to censoring is minor even for very strict communication rate constraints. We have also seen that the reductions in transmission rates are highest in the low SNR regime and in cases when the primary user is not present.

\section{ACKNOWLEDGMENT}

The authors would like to thank Prof. Saleem A. Kassam for very helpful discussions.

\section{REFERENCES}

[1] J. Lundén, V. Koivunen, A. Huttunen and H. V. Poor, "Spectrum Sensing in Cognitive Radios Based on Multiple Cyclic Frequencies," in Proc. of the Second Int. Conf. on Cognitive Radio Oriented Wireless Networks and Communications, Orlando, FL, USA, Jul. 31-Aug. 3, 2007.

[2] A. V. Dandawaté and G. B. Giannakis, "Statistical Tests for Presence of Cyclostationarity," IEEE Trans. Signal Process., vol. 42, no. 9, pp. 2355-2369, Sep. 1994.

[3] C. Rago, P. Willett, and Y. Bar-Shalom, "Censoring Sensors: A LowCommunication-Rate Scheme for Distributed Detection," IEEE Trans. Aerosp. Electron. Syst., vol. 32, no. 2, pp. 554-568, Apr. 1996.

[4] S. Appadwedula, V. V. Veeravalli, and D. L. Jones, "Robust and LocallyOptimum Decentralized Detection With Censoring Sensors," in Proc. of the Fifth Int. Conf. on Information Fusion, Jul. 8-11, 2002, pp. 56-63 vol. 1.

[5] R. S. Blum and B. M. Sadler, "A New Approach to Energy Efficient Signal Detection," in Proc. of the 41st Annual Conf. on Information Sciences and Systems (CISS), Baltimore, MD, USA, Mar. 2007.

[6] H. Bohman, "Numerical Inversions of Characteristic Functions," Scandinavian Actuarial Journal, pp. 121-124, 1975.

[7] 3rd Generation Partnership Project, "Technical Specification Group Radio Access Networks, Deployment aspects (Release 7)," $3 G P P$ specification: 25.943, 3GPP TR 25.943 V7.0.0, Jun. 22, 2007. [Online]. Available: http://www.3gpp.org/ftp/Specs/html-info/25943.htm. [Accessed Oct. 1, 2007]. 